\title{
Electrospray Micromixer Chip for On-Line Derivatization and Kinetic Studies
}

\author{
Mélanie Abonnenc, Loìc Dayon, † Brice Perruche, Niels Lion, and Hubert H. Girault* \\ Laboratoire d'Electrochimie Physique et Analytique, Ecole Polytechnique Fédérale de Lausanne (EPFL), Switzerland
}

An electrospray microchip for mass spectrometry comprising an integrated passive mixer to carry out on-chip chemical derivatizations is described. The microchip fabricated using UV-photoablation is composed of two microchannels linked together by a liquid junction. Downstream of this liquid junction, a mixing unit made of parallel oblique grooves is integrated to the microchannel in order to create flow perturbations. Several mixer designs are evaluated. The mixer efficiency is investigated both by fluorescence study and mass spectrometric monitoring of the tagging reaction of cysteinyl peptides with 1,4-benzoquinone. The comparisons with a microchip without a mixing unit and a kinetic model are used to assess the efficiency of the mixer showing tagging kinetics close to that of bulk reactions in an ideally mixed reactor. As an ultimate application, the electrospray micromixer is implemented in a LC-MS workflow. Online derivatization of albumin tryptic peptides after a reversed-phase separation and counting of their cysteines drastically enhance the protein identification.

Mass spectrometry (MS) based workflows play a major role in proteomics, ${ }^{1,2}$ to identify, characterize, and quantify proteins at an ever increasing throughput, and in ever more complex mixtures. A number of approaches have been investigated to complement the mass spectrometric analysis of tryptic peptides based on chemical tagging, in order to isolate specific subclasses of proteins by affinity baits, or for quantitation purposes. Furthermore, the use of nonquantitative addition of chemical probes to increase the information content of MS analysis is a promising approach. For example, Sechi and Chait proposed the differential alkylation of cysteine residues as a way to increase the identification score in peptide mass fingerprinting. ${ }^{3}$ Over the last years, our lab has been involved in the study of benzoquinone reagents as specific tags for cysteine residues in acidic solution. More particularly, a novel methodology based on the on-chip electrochemical tagging of cysteines, by oxidation of hydroquinone into benzoquinone at the microchip electrode, during ESI-MS of

* Corresponding author. E-mail: hubert.girault@epfl.ch. Laboratoire d'Electrochimie Physique et Analytique, EPFL-SB-ISIC-LEPA, Station 6, Ecole Polytechnique Fédérale de Lausanne, CH-1015, Lausanne, Switzerland. Fax: +41.21. 693.36.67.

$\dagger$ Current address: Biomedical Proteomics Research Group, Department of Structural Biology and Bioinformatics, Faculty of Medicine, University of Geneva, Switzerland.

(1) Aebersold, R.; Mann, M. Nature 2003, 422, 198-207.

(2) Domon, B.; Aebersold, R. Science 2006, 312, 212-217.

(3) Sechi, S.; Chait, B. T. Anal. Chem. 1998, 70, 5150-5158. peptides has been introduced. ${ }^{4}$ The potential of this on-line electrochemical tagging was demonstrated with microfabricated electrospray emitters, where instrumental parameters (current density at the electrospray electrode, residence time of analytes and chemical probes, etc.) can be properly tailored and controlled. However, this methodology was so far limited to off-line analysis of peptide fractions. In order to bring the potential of tagging methodologies to on-line analysis (e.g., in HPLC-ESI-MS analysis of tryptic peptides), we have developed here a combined derivatization/electrospray device where we physically mix the samples with the labeling reagent.

A number of studies have been performed to study chemical reaction kinetics by ESI-MS, pioneered by Lee et al..$^{5}$ In their setup, reactants were mixed manually in a vessel that was directly coupled to the ESI source. The time scale of these experiments was of the order of several seconds. Continuous-flow mixing setup and ESI-MS detection were used for various purposes, such as for the method of "time-resolved" ESI-MS ${ }^{6}$ to study protein folding ${ }^{7}$ and enzymatic reactions. ${ }^{8,9}$ Microreactors offer a good alternative to all these conventional laboratory-scale equipments, because they allow low reagent consumption and fast mixing as well as a continuous flow operative mode and real-time analysis. ${ }^{10}$ Reinhoudt's group introduced a nanoflow ESI interface (nESI) where a reactor chip, based on diffusion along a microchannel, was mounted on an ESI-MS nanoflow interface. ${ }^{11}$ Whereas there have been numerous reports about chip MS interfaces such as direct emitters, ${ }^{12-14}$ arrayed emitters, ${ }^{15,16}$ or HPLC-chip MS, ${ }^{17,18}$ there are

(4) Dayon, L.; Roussel, C.; Prudent, M.; Lion, N.; Girault, H. H. Electrophoresis 2005, 26, 238-247.

(5) Lee, E. D.; Mück, W.; Henion, J. D.; Covey, T. R. J. Am. Chem. Soc. 1989, 111, 4600-4604

(6) Konermann, L.; Collings, B. A.; Douglas, D. J. Biochemistry 1997, 36, 55545559 .

(7) Konermann, L.; Pan, J.; Wilson, D. J. BioTechniques 2006, 40, 135-141.

(8) Wilson, D. J.; Konermann, L. Anal. Chem. 2004, 76, 2537-2543.

(9) Liesener, A.; Karst, U. Anal. Bioanal. Chem. 2005, 382, 1451-1464.

(10) Brivio, M.; Verboom, W.; Reinhoudt, D. N. Lab Chip 2006, 6, 329-344.

(11) Brivio, M.; Liesener, A.; Oosterbroek, R. E.; Verboom, W.; Karst, U.; van den Berg, A.; Reinhoudt, D. N. Anal. Chem. 2005, 77, 6852-6856.

(12) Rohner, T. C.; Rossier, J. S.; Girault, H. H. Anal. Chem. 2001, 73, 53535357.

(13) Lazar, I. M.; Ramsey, R. S.; Jacobson, S. C.; Foote, R. S.; Ramsey, J. M. J. Chromatogr., A 2000, 892, 195-201.

(14) Figeys, D.; Lock, C.; Taylor, L.; Aebersold, R. Rapid Commun. Mass Spectrom. 1998, 12, 1435-1444

(15) Dethy, J. M.; Ackermann, B. L.; Delatour, C.; Henion, J. D.; Schultz, G. A. Anal. Chem. 2003, 75, 805-811.

(16) Dayon, L.; Abonnenc, M.; Prudent, M.; Lion, N.; Girault, H. H. J. Mass Spectrom. 2006, 41, 1484-1490.

(17) Yin, H.; Killeen, K. J. Sep. Sci. 2007, 30, 1427-1434.

(18) Yin, H.; Killeen, K.; Brennen, R.; Sobek, D.; Werlich, M.; van de Goor, T. Anal. Chem. 2005, 77, 527-533. 
few reports of microreactors directly coupled to the ESI-MS and even more directly integrated to the electrospray emitter microchip.

Because of the small dimensions of microchannels as well as the limited range of obtainable linear flow rates, the flow in microchannels is confined to the laminar regime and mixing is dominated by molecular diffusion. ${ }^{19}$ Micromixing may be accomplished using different approaches. Active mixing may involve either external energy sources such as electroosmotic flow, ${ }^{20,21}$ external pressure gradient, ${ }^{22}$ and electrokinetic instability ${ }^{23,24}$ to perturb fluid streamlines into a mixing state. Another approach may consist in passive micromixing based on the use of geometries within the microchannels to mix the fluids, ${ }^{25}$ such as the zigzag micromixer. ${ }^{26}$ One of the best-known designs of passive microfluidic mixing is the herringbone mixer..$^{27,28}$ The system uses a series of herringbone ridges placed on the bottom of the channel to create two counter-rotating vortices. To date, there have been a number of theoretical, experimental, and numerical studies aimed at the optimization of such micromixers. ${ }^{29,30}$ More particularly, in the case of the staggered herringbone micromixer (SGM), oblique grooves serve to transport fluid from the apex of the groove structure to the downstream edges of the microchannel. As a result, fluid near the top of the microchannels recirculates in the opposite direction, and an overall helical flow pattern is created.

The present study explores the application of a new design of electrospray emitter microchip with integrated oblique grooves constituting a passive mixing unit for on-line chemical modification of peptides and direct ESI-MS analysis. The polymer microchip is composed of a main feed microchannel supplying the analyte, linked by a liquid junction to a secondary microchannel for providing the tagging reagents. Downstream of the liquid junction, a mixing unit constituted of a series of photoablated oblique grooves allows the mixing of the two incoming flows. A carbon microelectrode in contact with the feed channel solution is also integrated to the microchip to apply the voltage to the solution and consequently induce the ESI process. First, the microfluidic device with the mixing unit was characterized by fluorescence imaging and compared to a similar device without any mixing unit in order to evaluate the efficiency of the mixing. In a second step, on-chip chemical tagging of cysteines with benzoquinone was performed on a model peptide containing three cysteines to illustrate the feasibility of the on-line tagging reaction directly on the electrospray microchip and to assess the performance of such

(19) Squires, T. M.; Quake, S. R. Rev. Mod. Phys. 2005, 77, 977-1026.

(20) Johnson, T. J.; Ross, D.; Locascio, L. E. Anal. Chem. 2002, 74, 45-51.

(21) Lin, J. L.; Lee, K. H.; Lee, G. B. Electrophoresis 2005, 26, 4605-4615.

(22) Dodge, A.; Jullien, M. C.; Lee, Y. K.; Niu, X.; Okkels, F.; Tabeling, P. C. R. Phys. 2004, 5, 557-563.

(23) Oddy, M. H.; Santiago, J. G.; Mikkelsen, J. C. Anal. Chem. 2001, 73, 58225832 .

(24) Sundaram, N.; Tafti, D. K. Anal. Chem. 2004, 76, 3785-3793.

(25) Hardt, S.; Drese, K. S.; Hessel, V.; Schönfeld, F. Microfluid. Nanofluid. 2005, 1, 108-118.

(26) Mengeaud, V.; Josserand, J.; Girault, H. H. Anal. Chem. 2002, 74, 42794286.

(27) Stroock, A. D.; Dertinger, S. K. W.; Ajdari, A.; Mezic, I.; Stone, H. A.; Whitesides, G. M. Science 2002, 295, 647-651.

(28) Stroock, A. D.; Dertinger, S. K.; Whitesides, G. M.; Ajdari, A. Anal. Chem. 2002, 74, 5306-5312.

(29) Lee, H. Y.; Voldman, J. Anal. Chem. 2007, 79, 1833-1839.

(30) Lynn, N. S.; Dandy, D. S. Lab Chip 2007, 7, 580-587.

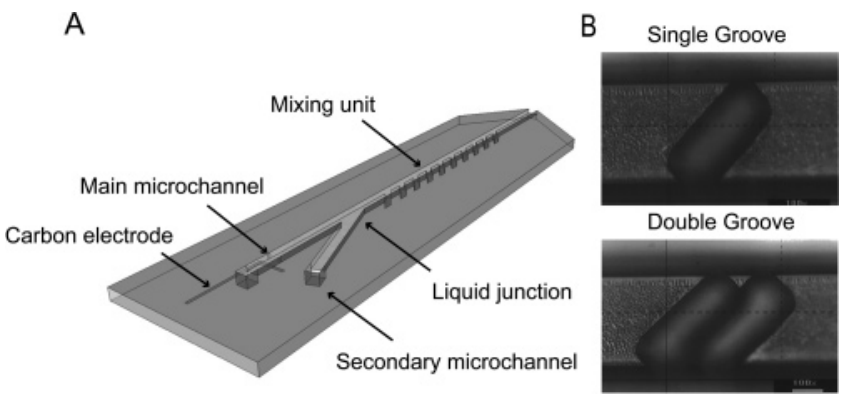

Figure 1. Electrospray microchip with a mixing unit. (A) Schematic representation of the device. (B) Microscopic view of the single and double grooves composing the mixing unit (top view). Typical dimensions of a single groove, $40 \times 100 \times 35 \mu \mathrm{m}^{3}$; double groove, $70 \times 100 \times 35 \mu \mathrm{m}^{3}$. The main and secondary microchannels are 6 and $2 \mathrm{~cm}$ long, respectively. The mixing unit is positioned at $3.5 \mathrm{~cm}$ from the main microchannel inlet. Grooves are photoablated on a $1.5 \mathrm{~cm}$ distance and are spaced by either 250 or $500 \mu \mathrm{m}$.

micromixers. Subsequently, the consecutive tagging on-chip of the three cysteines in the KCTCCA peptide was compared to an analytical kinetic model, showing a tagging kinetics close to that of bulk reactions in an ideally mixed reactor. Finally, on-line RPHPLC-tagging-ESI-MS of tryptic cysteinyl peptides of bovine serum albumin (BSA) was achieved with the electrospray micromixer chip.

\section{EXPERIMENTAL SECTION}

Chemicals. KCTCCA $\left(70 \%, M=627.8 \mathrm{~g} \mathrm{~mol}^{-1}\right)$ and Leuenkephalin (98.1\%, $M=555.6 \mathrm{~g} \mathrm{~mol}^{-1}$ ) were bought from Bachem (Bubendorf, Switzerland). 1,4-Benzoquinone (BQ) ( $\geq 98 \%, M=$ $108.1 \mathrm{~g} \mathrm{~mol}^{-1}$ ), fluorescein sodium, and acetic acid (>99.5\%) were supplied by Fluka (Buchs, Switzerland). Bovine serum albumin (BSA, $\geq 98 \%$ ) was purchased from Sigma (St. Louis, MO). Sequencing grade modified trypsin was from Promega (Madison, WI). Methanol ( $\geq 99.9 \%)$ and formic acid (FA) (>98\%) were from Merck (Darmstadt, Germany). Water (UV-HPLC grade) and acetonitrile (HPLC-gradient grade) were from Panreac Quimica S.A. (Barcelona, Spain). All chemicals were used without any further purification.

Device Microfabrication. The general ESI emitter microchip microfabrication process has been previously described in detail. ${ }^{12}$ The process for the device composed of two microchannels linked by a liquid junction is as follows (see Figure 1). First, an L-shaped microgroove was drilled on the backside of a polyethyleneterephthalate (PET) sheet of $100 \mu \mathrm{m}$ thickness (Melinex sheet from Dupont, Wilmington, DE) by photoablation with an $\mathrm{ArF}$ excimer laser (193 nm, Lambda Physik, Göttingen, Germany). After laser ablation, the chip was cleaned with $\mathrm{MeOH}$ and dried under nitrogen. Then, the L-shaped microgroove was filled twice with carbon ink to make the electrode. The chip was cured $1 \mathrm{~h}$ at $80^{\circ} \mathrm{C}$ and then laminated. In a second step, the main microchannel (100 $\mu \mathrm{m}$ wide, $6 \mathrm{~cm}$ long and $35 \mu \mathrm{m}$ depth) was photoablated on the topside of the microchip in such a way that the microchannel crossed the carbon electrode and revealed it.

To introduce the mixing unit, a smaller mask rotated by $45^{\circ}$ was used. Grooves $(40 \mu \mathrm{m} \times 100 \mu \mathrm{m})$ were made by regular static shots along a $1.5 \mathrm{~cm}$ long portion of the main microchannel and at the position of $3.5 \mathrm{~cm}$ from the main microchannel inlet, the number of static shots defining the groove depth (typically 100 

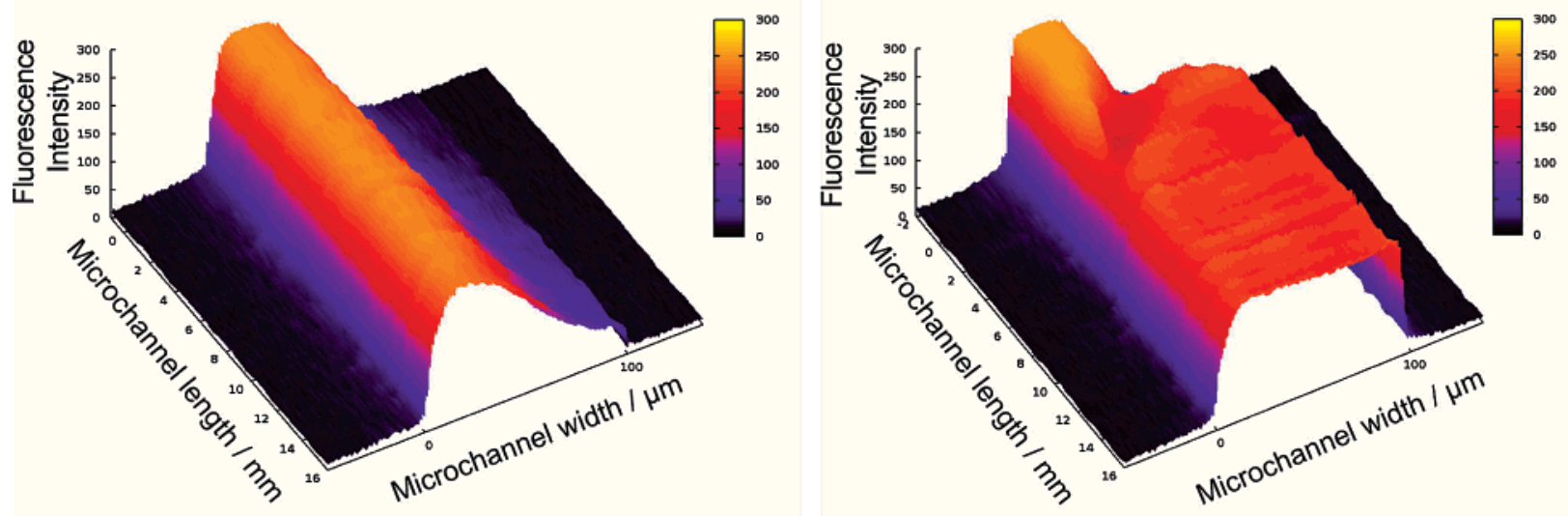

Figure 2. Fluorescence distribution along the microchannel $(A)$ without or $(B)$ with a mixing unit integrated to the microchip. When the mixing unit is integrated, single grooves are spaced by $250 \mu \mathrm{m}$. Flow rate $F_{\mathrm{V}}$ is $4 \mu \mathrm{L} \mathrm{min}^{-1}$ for the main microchannel and $1 \mu \mathrm{L}$ min ${ }^{-1}$ for the liquid junction providing the fluorescein.

static shots for a $35 \mu \mathrm{m}$ deep groove). Then, the microchip was rotated to drill the secondary microchannel $(100 \mu \mathrm{m}$ wide, $2 \mathrm{~cm}$ long, and $35 \mu \mathrm{m}$ depth). The microchannels were cleaned with $\mathrm{MeOH}$ and dried under nitrogen. Reservoirs were drilled at the channel extremities by static laser shots. The structure was sealed by lamination.

Mass Spectrometry. All experiments were performed on a LCQ DUO ion trap mass spectrometer (Thermo Electron, San José, CA). The heated capillary was kept at $200^{\circ} \mathrm{C}$. The microchip was positioned inside a homemade holder with two microholes for fluidic connections. The MS standard ESI interface was removed, and the holder was placed on a plate positioned on the probe slide adapter of the mass spectrometer.

The tagging experiments with KCTCCA were achieved with an ESI voltage of $3.5 \mathrm{kV}$ (positive ionization mode, $\mathrm{m} / \mathrm{z}$ of $500-$ $1000 \mathrm{Th})$. The HPLC-MS experiments were run with an ESI voltage of $3.7 \mathrm{kV}(\mathrm{m} / z$ of $150-2000 \mathrm{Th})$.

Fluorescence Imaging. The microchips were observed at working conditions with a microscope Axiovert 200 (Carl Zeiss, Gottingen, Germany) and a CCD-IRIS camera (Sony, Tokyo, Japan). Water was infused in the main microchannel at a flow rate $F_{\mathrm{V}}=4 \mu \mathrm{L} \mathrm{min}^{-1}$. A solution of fluorescein was provided from the secondary microchannel at $F_{\mathrm{V}}$ from 0 to $2 \mu \mathrm{L} \mathrm{min}{ }^{-1}$. The fluorescein distribution along the microchannel was observed under fluorescence. Pictures were taken before the mixing region and between each mixer groove (i.e., each $500 \mu \mathrm{m}$ ) on a total distance of $25 \mathrm{~mm}$. Imaging data were obtained both with microchips without and with a mixing area.

Image Analysis. The fluorescence intensity profile along the microchannel width section was extracted from images using Igor software (Wavemetrics, Portland, OR). For the same microchip all the fluorescence profile data were assembled using an in-house program written in $\mathrm{C}$ language, then interpolated, and plotted with Gnuplot 4.2 under Ubuntu Linux 7.04.

On-Chip Chemical Tagging of Cysteinyl Peptides. KCTCCA and $\mathrm{BQ}$ were diluted in $\mathrm{MeOH} / \mathrm{H}_{2} \mathrm{O} / \mathrm{AcOH}$ (50\%:49\%:1\%) at a concentration of $10 \mu \mathrm{M}$ and from 5 to $100 \mathrm{mM}$, respectively. KCTCCA was provided by the main microchannel at $F_{\mathrm{V}}=4 \mu \mathrm{L}$ $\min ^{-1}$. The $\mathrm{BQ}$ solution was infused in the chip from the liquid junction at various $F_{\mathrm{V}}$ from 1 to $2 \mu \mathrm{L} \mathrm{min}^{-1}$.

Simulations of the Multitagging of Cysteines in Peptides. The kinetic model was based on a set of differential equations that can be solved analytically. Calculations for each experimentally tested condition were run with Maple 9.5 (Waterloo Maple Inc., ON, Canada).

BSA Digestion and On-Line HPLC-Tagging-MS of Cysteinyl Peptides. An amount of $1 \mathrm{mg}$ of BSA was dissolved in 1 $\mathrm{mL}$ of ammonium bicarbonate buffer $(5 \mathrm{mM}, \mathrm{pH}=8)$. Then, 0.62 $\mathrm{mg}$ of dithiothreitol (to get a final concentration of $4 \mathrm{mM}$ ) and 20 $\mu \mathrm{g}$ of trypsin (protein ratio 1:50) were added. The digestion was run at $37{ }^{\circ} \mathrm{C}$ for $5 \mathrm{~h}$. An amount of $1 \mu \mathrm{g}$ of the BSA digest was separated on a LC Packings Ultimate (Dionex, Olten, Switzerland) capillary RP-HPLC system equipped with a UV detector (multimonitoring at 214, 250, and $280 \mathrm{~nm}$ ). An LC Packings C18 PepMap 100 column ( $15 \mathrm{~cm}, 3 \mu \mathrm{m}, 100 \AA$ ) was used. The separation was run for 60 min using a gradient of $\mathrm{H}_{2} \mathrm{O} / \mathrm{CH}_{3} \mathrm{CN} / \mathrm{FA} 98 \%: 2 \%: 0.1 \%$ (solvent $\mathrm{A}$ ) and $\mathrm{H}_{2} \mathrm{O} / \mathrm{CH}_{3} \mathrm{CN} / \mathrm{FA} 20 \%: 80 \%: 0.085 \%$ (solvent B). The gradient was run as follows: $0-3 \min 100 \% \mathrm{~A}$, then to $50 \% \mathrm{~B}$ at $40 \mathrm{~min}$, and $100 \% \mathrm{~B}$ at $50 \mathrm{~min}$ for $10 \mathrm{~min}$, at $F_{\mathrm{V}}=4 \mu \mathrm{L} \mathrm{min}^{-1}$.

The HPLC capillary outlet was directly connected to the microchip inlet using the holder system described above. The secondary microchip inlet was fed either by a sheath liquid solution $\left(\mathrm{H}_{2} \mathrm{O} / \mathrm{CH}_{3} \mathrm{CN} / \mathrm{FA} 50 \%: 50 \%: 0.1 \%\right)$ or the tagging reagent solution (i.e., $\mathrm{BQ}$ at a concentration from 5 to $100 \mathrm{mM}$ in $\mathrm{H}_{2} \mathrm{O}$ / $\left.\mathrm{CH}_{3} \mathrm{CN} / \mathrm{FA} 50 \%: 50 \%: 0.1 \%\right)$ at $F_{\mathrm{V}}=1 \mu \mathrm{L} \mathrm{min}{ }^{-1}$.

Protein Identification. Mascot version 2.2 (http://www.matrixscience.com) was used to perform the database interrogation. Mascot enables, in its "Sequence query" module, the specification of amino acid content such as cysteine residues. The search was performed in the UniProt-Swiss-Prot database (Swiss-Prot Release 54.8 of October 23, 2007, 287050 entries) and restrained to the Chordata taxonomy. Trypsin was specified as the enzyme with up to one missed cleavage allowed. No modification was entered. The peptide mass tolerance was fixed to $\pm 0.5 \mathrm{Da}$, and the peptide charges to $1+, 2+$, and $3+$. 


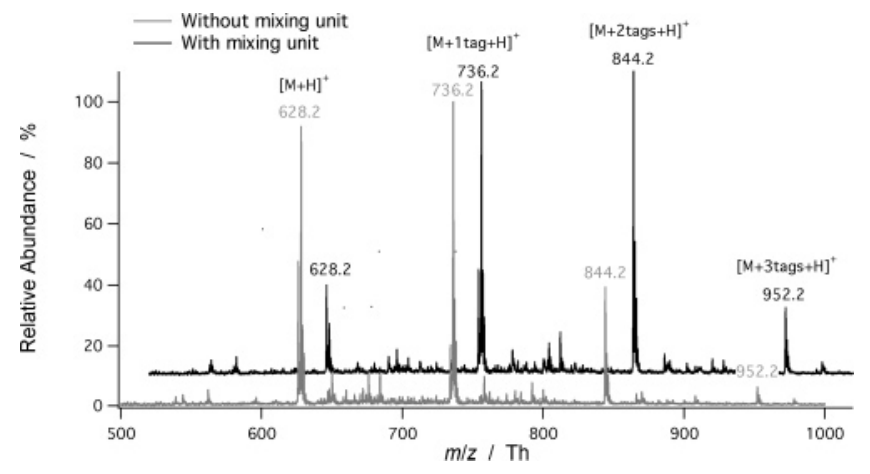

Figure 3. Mass spectra corresponding to the multitagging of KCTCCA with $B Q$ in an electrospray microchip with or without the mixing unit. KCTCCA $10 \mu \mathrm{M}$ was infused from the main microchannel at $4 \mu \mathrm{L} \mathrm{min}-1$ and $10 \mathrm{mM} \mathrm{BQ}$ from the liquid junction at $1 \mu \mathrm{L} \mathrm{min}-1$. The mass spectra were integrated over $4 \mathrm{~min}$ of monitoring.

\section{RESULTS AND DISCUSSION}

The ESI microchips studied here are typically made of a main microchannel with an embedded carbon band microelectrode and linked by a liquid junction to a secondary microchannel. The fabrication process is quite simple and based on UV-photoablation of polyethylene-terephthalate. Downstream of the liquid junction, a mixing unit made of photoablated parallel oblique grooves is integrated to create a flow perturbation along the microchannel as illustrated in Figure 1. The microchips were placed in a holder with capillary connections to fill in the two microchannels under flow-controlled conditions. The microchip was then positioned in front of the mass spectrometer, and the spray voltage was directly applied to the embedded carbon electrode.

The double microchannel electrospray device was first characterized with two different peptide solutions showing a good correlation between the flow rates applied in both microchannels and the respective MS ion count intensities (data not shown) ${ }^{31}$ The microchip geometry was shown to accommodate flow rates ranging from 1 to $7 \mu \mathrm{L} \mathrm{min}^{-1}$. In the present study, total flow rates from 4 to $6 \mu \mathrm{L} \mathrm{min}{ }^{-1}$ were applied, at which good spray stability was demonstrated (mean RSD of $4.95 \%$ ).

Mixing Efficiency with Different Groove Geometries. Figure 2 illustrates the fluorescence repartition in a smooth microchannel in the absence of a mixing unit (Figure 2A) and in a microchannel with photoablated oblique grooves (Figure 2B) along a $15 \mathrm{~mm}$ portion. In all cases, the main microchannel was infused with water at a flow rate $F_{\mathrm{V}}=4 \mu \mathrm{L} \mathrm{min}{ }^{-1}$. A fluorescein solution was provided from the secondary microchannel at $F_{\mathrm{V}}$ ranging from 0 to $2 \mu \mathrm{L} \mathrm{min}{ }^{-1}$. Under laminar flow conditions, diffusion and linear convection are the only two parameters acting on fluids. However, with flow rates in the microliter per minute range, diffusion alone is not sufficient to mix the species in a few centimeter long microchannel. Typically, in the present experimental conditions, a microchannel of tens of centimeters length would be required to accomplish the mixing of both solutions by diffusion only. Comparatively, with the devices including a mixing unit, the fluorescence inside the microchannel becomes homogeneous after only few millimeters from the beginning of the mixer that clearly demonstrates the advantages of the mixer for fast chemical reaction at such flow rates.

(31) Lion, N.; Gellon, J. O.; Girault, H. H. Rapid Commun. Mass Spectrom. 2004, 18, 1614-1620.
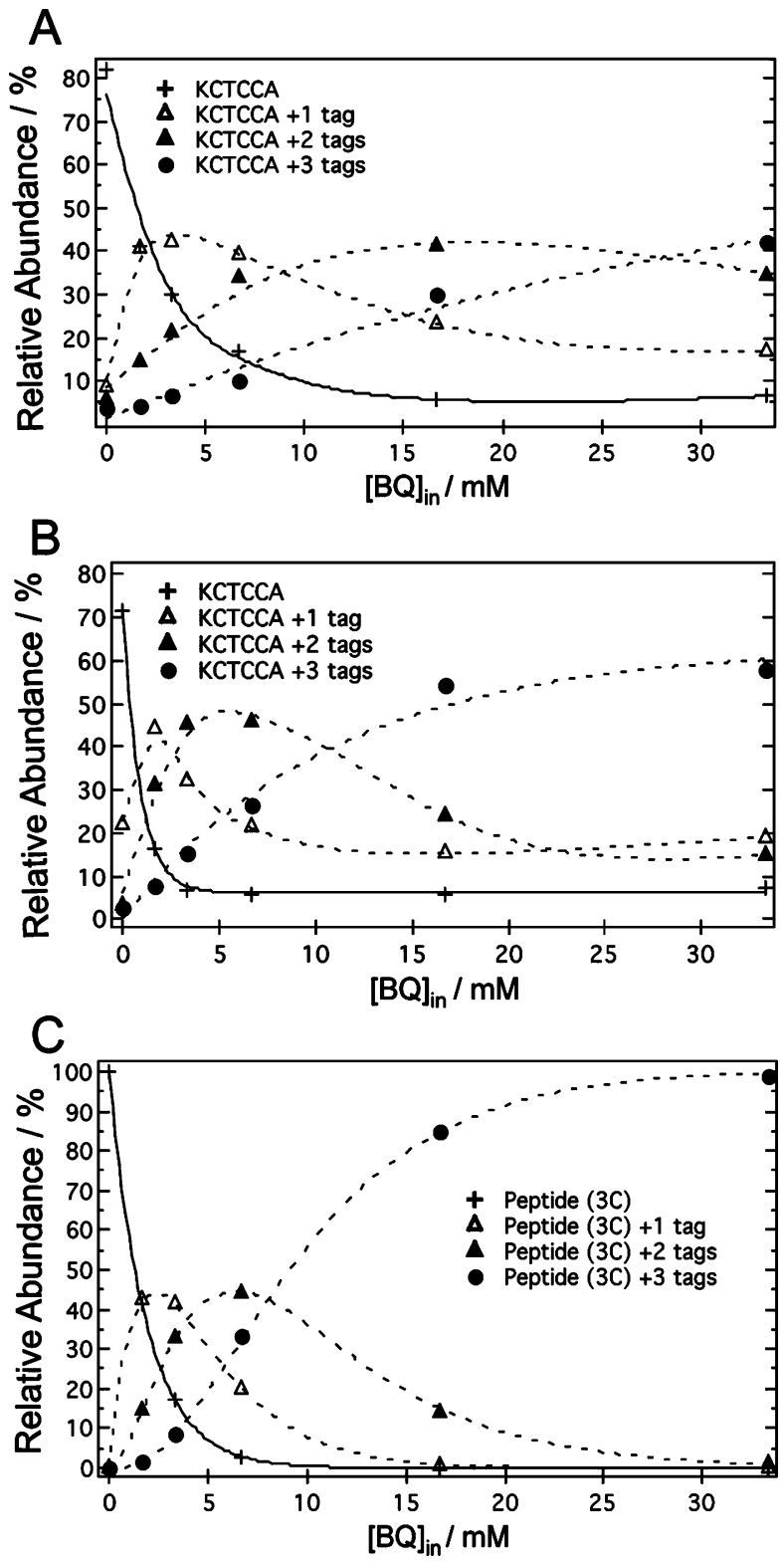

Figure 4. Relative abundance percentage of the intermediary multitagged KCTCCA products as a function of the $B Q$ concentration when using a microchip (A) without or $(B)$ with the mixing unit. In part $\mathrm{C}$ are represented the multitagged peptide distributions calculated from an analytical kinetic model for a bulk reaction. The dotted lines are the interpolation of data points to aid visualization. KCTCCA 10

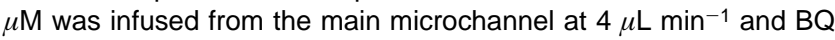
from the liquid junction with different concentrations at $F_{\mathrm{V}}=2 \mu \mathrm{L}$ $\mathrm{min}^{-1}$. The spectra were averaged over $4 \mathrm{~min}$ to determine the relative abundance of the tagged peptides.

The effects of the groove width, the intergroove distance, and the liquid flow rate were optimized by comparing the fluorescence distribution of four configurations of mixers, with single or double grooves spaced by 250 or $500 \mu \mathrm{m}$ (see Supporting information Figure S1). Single grooves are more efficient than double grooves probably due to a sharper flow perturbation. Moreover, by comparing the efficient mixing distance between mixers with grooves spaced by 250 and $500 \mu \mathrm{m}$, it appears clearly that closer grooves increase the flow spiraling, speeding up the mixing. In the literature, optimized groove geometries have been simulated, showing that large grooves and smaller intergroove distance 


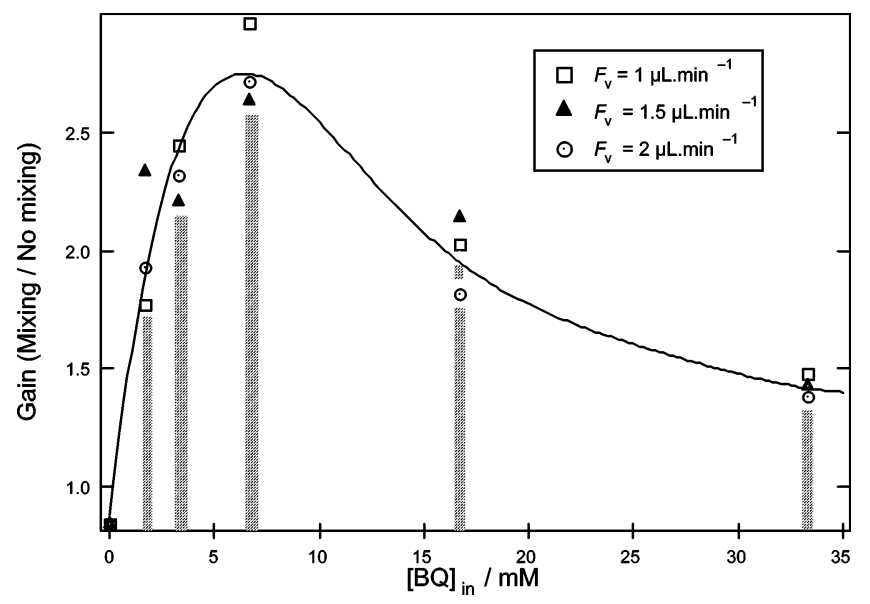

Figure 5. Representation of the gain when using a microchip with a mixer compared to without. The gain is calculated as the ratio of the fully tagged KCTCCA relative abundances, obtained with both microchips. KCTCCA $10 \mu \mathrm{M}$ was infused from the main microchannel at $4 \mu \mathrm{L} \min ^{-1}$ and $\mathrm{BQ}$ from the liquid junction with different

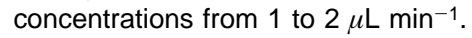

results in a higher magnitude of helical flow..$^{30}$ Because of fabrication constraints (available mask geometry and actual software control limitations), the polymer ESI microchip cannot present such optimal design. The alternative proposed here allows a sufficient mixing in the microchip geometry used. The flow rates applied do not affect the mixing efficiency as the total flow rate varies only from 4.5 to $6 \mu \mathrm{L} \mathrm{min}{ }^{-1}$. As a conclusion, the design with single grooves spaced by $250 \mu \mathrm{m}$ was the most efficient for this electrospray micromixer chip.

MS Evaluation of the Electrospray Micromixer for Chemical Modification of Peptides. The cysteine tagging in peptides with 1,4-benzoquinone (BQ) was compared when using microchips with and without integrated mixing units. With the typical flow rates and microchip dimensions used, the species residence time once mixed, from the liquid junction to the outlet, is around $1 \mathrm{~s}$. The reaction between the thiol group of cysteine and the $\mathrm{BQ}$ presents a reaction rate fast enough to be achieved on-line in the microchip..$^{32}$ Here, the KCTCCA peptide was infused from the main microchannel at a concentration of $10 \mu \mathrm{M}$ and $F_{\mathrm{V}}=4 \mu \mathrm{L}$ $\min ^{-1}$. The BQ was provided by the secondary microchannel at $F_{\mathrm{V}}$ from 1 to $2 \mu \mathrm{L} \mathrm{min}^{-1}$. Different initial concentrations of $\mathrm{BQ}$ $\left([\mathrm{BQ}]_{0}\right)$ were tested from 5 to $100 \mathrm{mM}$. Varying the flow rates modifies simultaneously the ratio of incoming concentrations between the peptide $\left([\mathrm{P}]_{\mathrm{in}}\right)$ and $\mathrm{BQ}\left([\mathrm{BQ}]_{\mathrm{in}}\right)$, and the reaction time (i.e., residence time) inside the microchannel. Figure 3 is a comparison of the distribution of tagged peptides in both microchips for $[\mathrm{BQ}]_{0}=10 \mathrm{mM}$. For such a concentration of tagging reagent, the reaction is not going to completion in $1 \mathrm{~s}$, thus allowing the detection and comparison of the reaction intermediates. In the absence of the mixing unit, the two most abundant species detected are the native peptide $(\mathrm{m} / z=628.2 \mathrm{Th})$ and the singly tagged peptide $(\mathrm{m} / z=736.2 \mathrm{Th})$. Comparatively, with the micromixer, the two most abundant peaks correspond to the singly tagged and doubly tagged peptides $(\mathrm{m} / z=844.2 \mathrm{Th})$. By increasing the encounter between the peptides and the tagging

(32) Roussel, C.; Rohner, T. C.; Jensen, H.; Girault, H. H. Chem. Phys. Chem. 2003, 4, 200-206. reagent, the passive mixing of flows significantly improves the tagging extent.

Comparative Study of the Reaction Extent without or with the Mixing Unit. Figure $4 \mathrm{~A}, \mathrm{~B}$ represents the evolution of the tags on KCTCCA as a function of $[\mathrm{BQ}]_{\text {in }}$ for a fixed $\mathrm{BQ}$ flow rate of $2 \mu \mathrm{L} \mathrm{min}^{-1}$. The relative abundance percentage of each species was calculated according to the following relationship:

$$
\text { Relative Abundance } \%=\frac{\mathrm{IC}_{\mathrm{S}, i}}{\sum \mathrm{IC}_{\mathrm{S}, i}} \times 100
$$

where $\mathrm{IC}_{\mathrm{s}}$ is the ion count for the peptide and $i$ the number of tags attached on the peptide ( $i=0,1,2$, or 3$)$.

As previously, the experiments were run with microchips without and with the mixer. The total flow rate in the microchannel was kept at $6 \mu \mathrm{L} \mathrm{min}^{-1}$, which corresponds to a reaction time of $0.82 \mathrm{~s}$ from the beginning of the mixing unit to the microchannel outlet. With an increase in $[\mathrm{BQ}]_{\text {in }}$, the reaction rate increases as illustrated in Figure 4A,B and the tagged species are formed more rapidly. When the mixing unit is present, the reaction efficiency is higher compared to the one obtained without. Also, when increasing $[\mathrm{BQ}]_{\text {in }}$ to $33.3 \mathrm{mM}$, the fully tagged peptide abundance finally reaches a plateau meaning that the reaction is close to completion. On a few millimeter long microchannel, the reaction extent is then exploitable at different stages varying the microchannel length, the flow rates, the concentration of each species, and the presence or performance of the mixer.

Comparative Study of the Reaction Extent with a Kinetic Model. An analytical kinetic model has been previously proposed to predict tagging extents at the end of a microchannel in an electrospray microchip. ${ }^{33}$ In the addition between $\mathrm{BQ}$ tags and a peptide containing one cysteine, the rate law follows a first-order kinetics for each reactant:

$$
v=-\frac{\mathrm{d}[\mathrm{BQ}]}{\mathrm{d} t}=-\frac{\mathrm{d}[\mathrm{P}]}{\mathrm{d} t}=\frac{\mathrm{d}\left[\mathrm{PQ}_{1}\right]}{\mathrm{d} t}=k[\mathrm{P}][\mathrm{BQ}]
$$

where $v$ is the rate of the reaction, $k$ is the rate constant, and $[B Q],[P]$, and $\left[\mathrm{PQ}_{1}\right]$ represent, respectively, the concentration of $\mathrm{BQ}$ tags, of a peptide $\mathrm{P}$ containing one cysteine residue, and of the tagged product $\mathrm{PQ}_{1}$ at the time $t$.

The kinetic model can be applied to consecutive reactions within the electrospray micromixer when the peptide possesses several cysteine units. In the case of a three-cysteine-containing peptide, the first step has an apparent rate constant that can be considered as $k_{1}=3 k$, since the rate law can be formulated as illustrated below.

$$
\mathrm{P} \underset{k_{1}=3 k}{\stackrel{\mathrm{BQ}}{\longrightarrow}} \quad \mathrm{PQ}_{1} \underset{k_{2}=2 k}{\mathrm{BQ}} \quad \mathrm{PQ}_{2} \underset{k_{3}=k}{\stackrel{\mathrm{BQ}}{\longrightarrow}} \mathrm{PQ}_{3}
$$

To simplify, this model assumes that (i) the reactivity for a cysteine in any peptide is the same as that of L-cysteine $(k=210$ $\mathrm{M}^{-1} \mathrm{~s}^{-1}$ ) and (ii) all the cysteines have the same reactivity. Moreover, the concentration of $\mathrm{BQ}\left([\mathrm{BQ}]_{\mathrm{in}}\right)$ used here was in

(33) Dayon, L.; Roussel, C.; Girault, H. H. J. Proteome Res. 2006, 5, 793-800. 

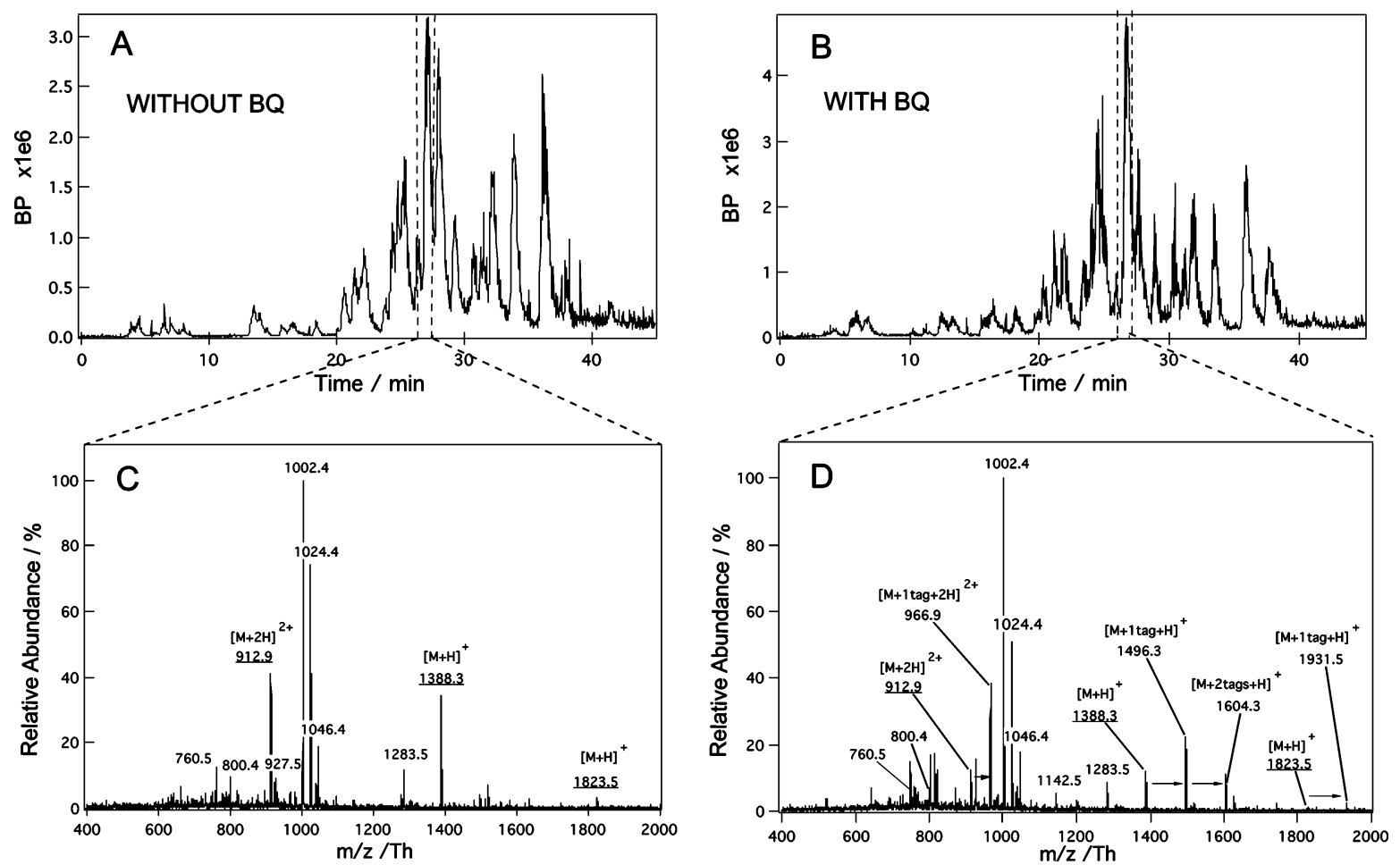

Figure 6. On-line chemical derivatization of cysteinyl peptides of a BSA digest. Parts $A$ and $B$ represent the base peak chromatograms of the LC-MS analysis of the BSA digest with a microchip infused by a solution containing or not containing the $B Q$ reagent $\left([B Q]_{0}=50 \mathrm{mM}\right)$, respectively. The mass spectra were integrated over $1 \mathrm{~min}$ on both chromatograms at the same elution time. Parts $C$ and $D$ present the integrated mass spectra in the absence or presence of $B Q$, respectively. Sequences of the cysteinyl identified peptides: $[\mathrm{M}+\mathrm{H}]^{+}=1388.3 \mathrm{Da}$ "EYEATLEECCAK"; [M + H] $]^{+}=1823.5$ Da "RPCFSALTPDETYVPK". The main microchannel was connected to the HPLC flow outlet (4 $\mu$ L $\min ^{-1}$ ). The solution provided by the liquid junction (without or with $\mathrm{BQ}$ ) was kept at $1 \mu \mathrm{L} \mathrm{min}^{-1}$.

excess compared to that of the cysteines. Typically, $[B Q]_{\text {in }}$ was at least 20 times higher than that of total cysteines. Therefore, the concentration of $\mathrm{BQ}$ can be assigned to be constant, simplifying the resolution of the kinetic equations.

Figure $4 \mathrm{C}$ summarizes the theoretical calculation of the tagging extent on a three cysteine-containing peptide as a function of $[B Q]_{\text {in. }}$. The goal of these calculations is to evaluate the efficiency of the mixer compared to an analytical model where the species are assumed to be ideally mixed in solution. The comparison of parts A-C of Figure 4 clearly indicates that the kinetic behavior when species are flowing through a mixing unit approaches that of a bulk reaction. The small differences compared to the model are due to the main assumption made (i.e., the same reactivity of the different cysteines in the peptides and this, for each stage of the consecutive reactions) and to the ESI-MS process (i.e., the ion count for each species does not directly relate to their concentration because the presence of the quinone tag on the cysteines modifies their ionizability ${ }^{33}$ ). (Distributions for BQ flow rates of 1 and $1.5 \mu \mathrm{L} \mathrm{min}^{-1}$ are presented in Supporting Information Figure S2).

Evaluation of the Gain when Using the Electrospray Micromixer. The gain was evaluated by comparing the relative abundance of the fully tagged species in both microchips assuming that the objective is to get reaction completion. The gain is then expressed according to the following relation:

$$
\text { Gain }=\frac{\% \mathrm{PQ}_{3, \text { Mixing }}}{\% \mathrm{PQ}_{3, \text { NoMixing }}}
$$

where $\% \mathrm{PQ}_{3}$ is the relative abundance of the fully tagged peptide containing three cysteines. The gain was calculated for different concentrations and flow rates of $\mathrm{BQ}$ (Figure 5). As the curve fluctuations are similar for all the flow rates tested, a mean curve of the gain as a function of $[\mathrm{BQ}]_{\text {in }}$ was calculated in order to clearly show the benefits of the electrospray micromixer. In the presence of the micromixer, the minimal gain value is 1.5 when $[\mathrm{BQ}]_{0}<5$ $\mathrm{mM}$ or $[\mathrm{BQ}]_{0}>50 \mathrm{mM}$, meaning that the tagging reaction extent presents a $50 \%$ enhancement. It reaches $175 \%$ for $[\mathrm{BQ}]_{0}=20 \mathrm{mM}$.

On-Line HPLC-Chip-MS Derivatization of Tryptic Cysteinyl Peptides of BSA. The electrospray micromixer was hyphenated to an HPLC-MS workflow by directly connecting the HPLC capillary outlet to the main microchannel. The secondary microchannel was infused either by a sheath liquid or a $B Q$ solution at different concentrations of $10,20,50$, and $100 \mathrm{mM}$. The BQ infusion flow rate was kept at $1 \mu \mathrm{L} \mathrm{min}^{-1}$ in the following study because it presents the best compromise between spray stability and reasonable sample dilution. Figure 6 compares two LC-MS analyses of the BSA digest using the same microchip infused with a sheath liquid providing or not providing the $\mathrm{BQ}$ reagent. A single microchip was infused during several hours without apparent diminution of the spray quality or memory effect and showed good reproducibility for successive analyses as shown by the monitored base peak chromatograms of Figure $6 \mathrm{~A}, \mathrm{~B}$. The band broadening effect (peak width at half-maximum of a selected $\mathrm{m} / \mathrm{z}$ ) as well as the peak asymmetry (at $10 \%$ of maximal height) was evaluated in the HPLC-chip-MS workflow and compared to 
an HPLC-MS analysis with a classical ESI source. The details of the calculations are presented in Supporting Information Figure S3. Slight band broadening and peak tailing were noticed, due to the sum of the variance induced by a dead volume at the capillary connection, the on-chip liquid junction, and the mixing unit, but without significantly affecting the resolution of the separation and the detection.

For protein identification by peptide mass fingerprinting (PMF) including cysteine content, two ways of analysis are possible: (i) to carry out a single run with a BQ concentration at which both the untagged peptides and the tagged species are present. It necessitates careful control of the right concentration of $\mathrm{BQ}$ according to those of the eluted peptides. Figure 6B,D figures out averaged mass spectra at the same elution time obtained from both experimental conditions (without and with BQ). During this time window, two cysteinyl peptides were eluted: a one-cysteinecontaining peptide present as singly and doubly charged $(\mathrm{m} / z=$ 912.9 and $1823.5 \mathrm{Th}$ ) and a singly charged two-cysteine-containing peptide $(m / z=1388.3 \mathrm{Th})$. When $[\mathrm{BQ}]_{0}=50 \mathrm{mM}$ was infused in the microchip, the cysteinyl peptides present adducts of $\mathrm{BQ}$ $(+108 \mathrm{Da})$ with all the intermediary tagged species represented allowing the cysteine counting on a single spectrum. (ii) To perform two successive runs, one without and one with $B Q$ in large excess $\left([\mathrm{BQ}]_{0}=100 \mathrm{mM}\right)$, to fully tag the peptides (see Supporting Information Figure S4). The two peak lists are then compared to determine mass shifts and deduce cysteine content. As the hydrophobicity properties of the peptides are modified by the $\mathrm{BQ}$ tags, ${ }^{34}$ a postcolumn derivatization has the advantage not to modify the native peptide retention time that helps for easy and unmistaken peak list comparison and protein identification. ${ }^{35}$ The main inconvenience remains the need to perform two analyses that are both sample- and time-consuming.

In the present study, both techniques have been assessed using the electrospray micromixer chip for postcolumn cysteine tagging and showed comparable counting efficiency. The cysteinyl peptides identified by a single HPLC-chip-MS run, at $[\mathrm{BQ}]_{0}=50 \mathrm{mM}$, are recapitulated in Supporting Information (Table S5). The database search with Mascot was performed with the mass alone of all the identified peptides (control score) and with the addition of the peptide cysteine content. In both cases, the BSA was in the first position among all the identified proteins. With the peptide mass alone, the BSA was identified with a protein sequence coverage of $78 \%$ and a score of 50 that does not reach the confidence score (score $>72$ ). As expected, ${ }^{3}$ when the number of cysteines was indicated, the protein score reaches the value of 258 that definitively assures the confidence in its identification (complementary data about the on-line tagging of the BSA digest and the Mascot database search are given in Supporting Information Figure S6).

\section{CONCLUSIONS}

A polymer electrospray microchip with a mixing unit has been designed based on polymer UV-photoabalation. The mixing unit composed of a series of parallel oblique grooves along a portion of a channel is easily processed. By optical characterization of

(34) Dayon, L.; Girault, H. H. Anal. Bioanal. Chem. 2007, 389, 841-849.

(35) Strittmatter, E. F.; Kangas, L. J.; Petritis, K.; Mottaz, H. M.; Anderson, G. A.; Shen, Y.; Jacobs, J. M.; Camp, D. G., II; Smith, R. D. J. Proteome Res. 2004, 3, 760-769. the mixing, single grooves $40 \mu \mathrm{m}$ wide and $35 \mu \mathrm{m}$ deep, spaced $250 \mu \mathrm{m}$ apart were determined to provide the best mixing.

As a demonstration of efficiency, on-chip derivatization of cysteines in peptides was studied. KCTCCA peptide and benzoquinone reagent were provided by the main and secondary microchannels, respectively. The mixing unit clearly improves the reaction yield, approaching results obtained from reaction in the bulk condition. Furthermore, this on-chip chemical tagging reaction was implemented as postcolumn derivatization in an $\mathrm{LC}-$ MS analysis of tryptic peptides of BSA to determine their cysteine content. Other on-chip derivatization of (bio)molecules can also be envisaged, the only requirement being that the reaction rates should be fast enough to allow the reactions to reach completion within the short residence times in the electrospray micromixer.

Coupling the LC-MS setup with a micro- or nanoelectrospray emitter interface can present several benefits such as better sensitivity and the possibility to avoid the use of a sheath gas. Additionally, the electrospray micromixer introduced herein can prove useful in delivering an appropriate sheath liquid at the beginning of the RP-HPLC run, thus probably allowing the detection of the most hydrophilic compounds.

MS-based techniques have shown great promise in the area of chemical and biochemical kinetics. ESI-MS, in particular, has enormous potential as an alternative to the traditional methods for monitoring enzyme kinetics, because the reaction mixture can be infused directly into the ion source for on-line analysis while the reaction occurs in solution. Up until now, the major part of the kinetics studies by ESI-MS reported in the literature was achieved by mixing the reactants prior the ionization source. Such a microchip with a mixing unit presents a great potential for chemical kinetics studies as the reaction occurs on-chip just before MS. By control of the microchannel length and/or flow rate, the reaction time can be easily adjusted to investigate the reaction intermediates and products.

\section{ACKNOWLEDGMENT}

Jacques Josserand and Valérie Devaud are thanked for fruitful scientific conversations. This work was supported by a Swiss National Science Fundation grant entitled "Development for new analytical tools for proteomics” (Grant No. 200020-105489).

\section{SUPPORTING INFORMATION AVAILABLE}

Mixing efficiency depending on groove design constituting the mixing unit, distributions of the tagged products as a function of $[B Q]_{\text {in }}$ and their corresponding kinetic model calculations, band broadening and peak asymmetry in HPLC-chip-ESI-MS analysis, base peak and mass spectrum of on-line derivatization of typtic BSA peptide by HPLC-chip-MS with $[\mathrm{BQ}]_{0}=100 \mathrm{mM}$, identification of BSA cysteinyl peptides by on-chip derivatization, and online tagging of BSA tryptic cysteinyl peptides (UV chromatogram, base peak, submitted peak lists, Mascot scores). This material is available free of charge via the Internet at http://pubs.acs.org.

Received for review December 20, 2007. Accepted February 19, 2008.

$\mathrm{AC} 800058 \mathrm{H}$ 